\title{
Intelligent Systems and the Systematization of Pharmacological Knowledge: Application of the In Vitro Retina Model
}

\author{
Vera Maura Fernandes de Lima ${ }^{1,2}$, José Airamir Padilha de Castro $^{3,4}$ and Wolfgang Hanke *,5 \\ ${ }^{I}$ Federal University of São João del Rei (UFSJ)-Campus Divinopolis, Medical School, MG, Brazil \\ ${ }^{2}$ Experimental Surgery Laboratory-LIM 26, School of Medicine, University of São Paulo Av Dr Arnaldo 455 São Paulo, \\ SP, Brazil \\ ${ }^{3}$ ANVISA (Federal Agency for Public Health), Brazil \\ ${ }^{4}$ Special Adviser to the Public Health Secretary of the Federal Distric Government, Brasilia, DF, Brazil \\ ${ }^{5}$ University of Hohenheim, Inst. Physiology, Stuttgart, Germany
}

\begin{abstract}
In this century, the IT technology will spread intelligent systems based on cameras, sensors and computers across all fields of the human endeavor. The systematization of knowledge will reach new levels of organization and applications. Scientific knowledge will not be an exception. In this paper we present the results of validation tests made with the in vitro retina model in order to propose the systematization of pharmacological knowledge in experimental platforms supplied with data banks. Data banks can be made either for the speeding up of pre-clinical research or for the control of quality of pure substances or pharmaceutical preparations. These applications and other implementations of using the retinal spreading model in pharmacological research will be useful for the users, manufacturers and authorities of regulation of public health.
\end{abstract}

Keywords: Retinal spreading depression, pharmacology, quality control, generica, Brazil.

\section{INTRODUCTION}

Looking for systems to test the quality of pharmaceuticals it is obvious that these exist on the basis of cellular systems and on the level of animals in toxicological tests. Besides, high level biochemical diagnostics are available. However first, there is no adequate system on the tissue level, and second the presented systems are either slow, expensive or cannot be used on ethic reasons. The isolated retina method is fast, sensitive and less expensive than the existing models.

The creation of an IT platform to be used for pre-clinical drug tests or in quality control of pharmaceutics is feasible on two grounds. First, a similar platform controlled by telemetry has been made for rocket experiments, in which eight retinas could be observed and stimulated in a fully automatized set-up [1,2], laboratories using such platforms can collaborate easily among themselves and with regulatory agencies on-line. Second, there is considerable experimental knowledge to be systematized and shared already in place. For example, with respect to cholinergic pharmacology [3], molecular biology data and pharmacodynamic data can be put on the same data bank and any candidate compound performance in the system compared to previous results [3, 4]. In Brazil, from the sixties to the early nineties of the $X X$ century, several theses have been concluded using the

*Address correspondence to this author at the Universität Hohenheim, Instut für Physiologie - 230, Garbenstrasse 30, 70599 Stuttgart, Germany; Tel: 0049711459 22800; Fax: 004971145923736 ;

E-mail: wolfgang.hanke@uni-hohenheim.de in vitro retina model and this knowledge can be made available in the memory bank, for example the comparative behavior in vitro of the chick retina and several mammalian retinas [5].

What makes the in vitro retina model suitable for the creation of pharmacodynamic data banks are the remarkable intrinsic optical signal (IOS) of excitation waves and excitotoxic tissue responses. These macroscopic signals can be recorded non-invasively at several scales of space and time. For example, the temporal evolution of the IOS can be recorded as optical profiles. In Brazil, research on these optical profiles of in vitro chick retina began in the early sixties [6,7] and thus there is a considerable amount of acquired knowledge about the subjacent mechanisms associated with the generation of the IOS [8,9]. The applied research using the chick retina in pre-clinical drug development by one of the authors began in 1989, and through the years several protocols have been developed for the European and USA industries at the Membrane Physiology Division of the Hohenheim University. In preclinical research, the retina model can be applied for fast screening of CNS activity of a given compound [11], for the comparative modulation of excitability at intact tissue level (good for assessment of anti migraine and antiepileptic drugs) and to measure the protection against excitotoxicity of a given compound. From the pharmacological point of view, the central grey matter of retinas has all the relevant receptors families (cholinergic, dopaminergic, serotonergic etc.) for cortex and for heart, a major aim of interest in drug development [10]. Besides the IOS, electrophysiological wave concomitants have been used in pharmacological 
protocols either alone or together with the IOS or other concomitants [11].

The importance of such experimental platforms for Brazil and countries similar in industrial development comes from the size of the market and its particularities. The prescription drugs market was R\$ 36.7 billions in 2010 (US\$ 21.6 billions) with a growth of $9.9 \%$ within the year. The growth has been in two digits in the last five years. However, $85 \%$ of active principles in pure form are imported (the balance of payments deficit in these compounds was 3.4 billion in 2010) [LAFIS evaluation of pharmacological industry in Brazil, 12]. There are approximately 400 pharmacological industries in Brazil with 20 transnational companies responsible for $80 \%$ of the market $[13,14]$. These global companies never invested in research in Brazil or in industrial plants for their original substances. Also, the majority of the national companies import their substances to sell generic or similar presentations of original medicines. It is in the interest of the federal government for the country to dominate the innovation cycle for new medicines and at the same time to control the quality of generics and similar medicines (the government is the big buyer in this market because of the constitutional right of all citizens to free medicines). In Brazil, ANVISA (National Agency for Sanitary Vigilance) defined generic drugs as having bioequivalence with the brand name preparation (Federal Law 9787 of February 1999), defining bioequivalence in the same sense used by the Food and Drug Administration of the USA: if a pair of drugs have the same bioavailability, they are said to be bioequivalent. In both countries, the concept is used for the registration of generics. The proposed platform can generate a data bank to test the medicines after registration and is able to do so in a batch by batch basis. As late as 2004, 11 people died in Brazil poisoned by barium carbonate that contaminated the contrast Celobar ${ }^{\mathrm{R}}$ (barium sulfate). Due to exchange rate volatility, one enterprising chemist in an industry had tried to substitute the imported barium sulfate by homemade one.

The other application of a pharmacodynamic data bank is in pre-clinical research to help in speeding up some of the steps involved in the development of a new compound. Thus, a simple, fast, robust and cost effective method can be very useful in Brazil either as a tool to improve P\&D of national companies or as an improvement in the quality control of medicines.

To validate the retina model we used the simplest protocol that uses the IOS of retinal spreading depression waves (RSDs): it measures the spread velocity of the wavefront in control situation and under the influence of a drug. We compared the system response to European and Brazilian standards of pure substances to the ones furnished by companies, and also the response to the pure substance to the substance extracted from pills. The retina model performance was compared to HPLC responses to the same substances. Finally, a comparison of formulations was made for some preparations of Atenolol sold in Brazil as generic or similar to the AstraZeneca preparation.

\section{METHODS}

Animals: For the experiments chicken aged from 7 to 21 days were used. The use of animals in this study was approved at institutional level and in accordance with national and international regulations. At the beginning of each experiment, two animals were killed by decapitation and the eye cups were excised from the eye sockets.

Eyecup preparation: The eye cups were cut at the equator and the vitreous humor removed with tweezers, then they were transferred to plastic Petri dishes and covered with the standard Ringer solution for chick retinas.

Retina Ringer solution: The standard solution used in the experiments had the following composition: $100 \mathrm{mM}$ $\mathrm{NaCl}, 6 \mathrm{mM} \mathrm{KCL}, 1 \mathrm{mM} \mathrm{MgSO} 4,1 \mathrm{mM} \mathrm{CaCl} 2.2 \mathrm{H}_{2} \mathrm{O}, 1 \mathrm{mM}$ $\mathrm{NaH}_{2} \mathrm{PO}_{4}, 30 \mathrm{mM} \mathrm{NaHCO} 3,10 \mathrm{mM}$ Tris and $30 \mathrm{mM}$ glucose. The $\mathrm{pH}$ is adjusted with $\mathrm{HCl}$ to 7.4.

Setup: The setups are mounted on vibration-damped tables. They consist of an aluminium plate with four hollows for the Petri dishes, a heating pad, a perfusion system containing two four-channel peristaltic pumps with tube system and either one movable camera mounted on a motor driven carriage or 4 fixed cameras. The solution is slowly perfused $(1 \mathrm{ml} / \mathrm{min})$ with laminar flow. The data from the experiments were either stored on videotapes or DVD recorder disks.

Substances: Amlodipine Besilate EP, batch number $\mathrm{AB} / 002 / 3021$, was furnished by Biolabfarma from São Paulo, Brazil together with two boxes of tablets of the oral presentation Pressat ${ }^{\circledR} 10 \mathrm{mg}$ Lot 43679 and $5 \mathrm{mg}$ Lot 312909. The tablets used the lot $\mathrm{AB} / 002 / 3021$ as active substance. Amlodipine certified as the European Reference Substance, Lot 383.06.03.02 was acquired from MIKROMOL GmbH (Im Biotechnologiepark- TGZ II D14943 Luckenwalde, Germany). Atenolol certified as the Brazilian reference substance was furnished by the National Institute for the Quality Control of Health (INCQS) of FIOCRUZ, Rio de Janeiro, Brazil (declared purity of 99.99\%), Atenolol, racemic mix, and the enantiomers R (+) and L(-) laboratory grade (declared purity of 99,95\%), were acquired from Sigma-Aldrich Chemie $\mathrm{GmbH}$ (Steinheim, Germany). Three lots of Atenolol were donated by AstraZeneca, São Paulo, Brazil. Atenolol 25 mg tablets (Atenol®) and Atenolol $25 \mathrm{mg}$ tablets generic oral preparations from Biosintética, São Paulo, and EMS, Campinas, Brazil were bought from the pharmacy of the Pharmaceutical Sciences Faculty of São Paulo University. We simply bought the reference substance Atenol and the two generics that were avaiable at the university pharmacy at that moment. Sodium dodecyl sulfate (sodium lauryl sulfate) or SDS was acquired from Sigma-Aldrich Chemie GmbH.

Extraction of active principle from tablets: The tablets were smashed in a mortar and then the mortar was washed with 1 to $4 \mathrm{ml}$ of DMSO, depending on the bulk of the pills. The extract was then centrifugated at 4000 r.p.m to separate the soluble part from the rest. The DMSO solution was covered with standard Ringer solution to a volume of $500 \mathrm{ml}$. DMSO addition to the Ringer solution, up to $1 \%$ in volume does not alter the optical properties of the retinas or propagation velocity of RSD waves.

Chemical assays: A colorimetric assay was used to determine the SDS content of the pills- SDS was used in all Atenolol generics tested as a dispersant to improve tissue availability-. The Spectroquant cell test for anionic 
surfactants from Merck (1.14697.0001) was used for this purpose together with a spectrophotometer from Zeiss. Data were then recalculated in $\mathrm{mg} / \mathrm{pill}$ of total anionic surfactants under the assumption that no other anionic surfactants than SDS were present in the tablets (according to the list of ingredients attached to each generic), thus the data were representing the nominal SDS concentration. All Atenolol standards were tested in an HPLC system for purity. An ÄKTAexplorer system from Amersham Biosciences was used for this purpose in the analytic mode together with the Unicorn software and a "RP60 select B" column.

Additionally, tablets were controlled by HPLC analysis for their absolute Atenolol content (recalculated in $\mathrm{mg} / \mathrm{pill}$ ), again using the ÄKTA system.

Statistics and graphs: The graphs and the statistics were made using the GraphPad PRISM software from GraphPad Software Inc. and Origin from Microcal Software, Northampton, USA.

Protocol: Four retinas were measured in parallel in both setups. The waves were elicited mechanically by gently touching the retina with a fine tungsten electrode. Velocity was calculated from the time the wave front needed to travel over a defined distance on the monitor. After each elicited wave the temperature was controlled and maintained at $29^{\circ} \mathrm{C}+/-1{ }^{\circ} \mathrm{C}$. A minimum of 20 minutes interval was observed between stimulations in each retina. In each experiment, the four retinas were submitted to eight stimulations in sequence with a minimum of 20 minutes interval. Two controls waves, four under the perfusion with the drug, and two post-control waves during washing over with standard Ringer were recorded. The typical total recording time thus was about four hours. The change in the solution was slow $(1 \mathrm{ml} / \mathrm{min})$ throughout the experiment in order to maintain laminar flow avoiding optical disturbances in the system. The temporal evolution of the spread velocity of RSD waves gave a characteristic sequence expected for each drug. In Fig. (2), a flow diagram summarizes the protocol.

\section{RESULTS AND DISCUSSION}

The RSD IOS and the modulation of the spread velocity- In Fig. (1A, B) the IOS of a spreading wave is shown either as a sequence of frames or as the temporal evolution of optical changes within the tissue in the optical profile.

The RSD wave from the thermodynamic point of view is a wave in an excitable medium, and the frames show the whole medium displaying quiescent state (in front of the RSD), excited state (at the wavefront) and refractory state (behind the wavefront). Refractoriness ranges from the absolute to relative until at the end of the optical profile when the tissue is back to quiescence, ready to be excited again [15]. The temporal evolution of the optical change displayed in the optical profiles provides information about the system excitability: the profile has two peaks of increased light scatter and the interpeak interval (about 3 minutes at 30 degrees) the end of which represents the end of the absolute refractoriness. Within this interval, the tissue does not respond to any type of stimulation. Just after the absolute refractory state, the spread velocity is about $45 \%$ slower than control values $[16,17]$. The relative refractoriness duration coincides with the optical profile duration, and at the end of the second peak, the tissue susceptibility to stimulation is back to the quiescent state before stimulation. The same is true for the spread velocity; for example, with 10 minutes of interval between two consecutive waves, the spread velocity is about $75 \%$ of the control value. Thus, the maximum effect a compound can have, is a reduction of $50 \%$ in spread velocity (it is typical that with four retinas in parallel, at least one will not respond to stimulation while the other three will show waves with spread velocity reduced by $50 \%$ ) and this value is an indication that this drug brings the system close to absolute refractoriness, while a $25 \%$ reduction is equivalent to relative refractoriness of 10 minutes.

The stability of the system response within the applied protocol- Fig. (2) shows the experimental protocol. In Fig. (3), the stability of the system response is demonstrated. Four retinas are recorded in parallel and RSD waves were mechanically elicited every 30 minutes. The spread velocity was normalized with the mean among the 4 retinas for the first spread velocity representing $100 \%$ value. Each point is the mean of the four retinas and the standard deviation is shown for each mean. The results of two experiments are superimposed. Note the stability of the retinas response during both experiments.

The calcium channel antagonist Amlodipine effects and the system sensibility- The calcium antagonist Amlodipine is sold in Brazil in oral preparations called Pressat $^{\mathrm{R}}$, similar to the reference compound. The pure substance test was imported as a complex Amlodipine besilate. The Amlodipine besilate complex is used in the oral presentations of the drug due to a better stability at higher temperatures; the European standard Amlodipine used as reference had to be maintained at $8^{\circ} \mathrm{C}$ until use. We compared the system response to Amlodipidine besilate to the secondary European standard Amlodipine. Details of suppliers and extraction of principle from pills have already been given in the methods section.

In Fig. (4) we show the results of two experiments (four retinas each) at $70 \mu \mathrm{M}$ concentration comparing the effects of Amlodipine besilate with pure Amlodipine. The results are presented in non-normalized (upper part) and in normalized velocity spread form (lower part). Each point is the mean of the data of 4 retinas and the bar the SD for each value. The mean of the first two controls is used as the first value and the sequence of the waves two to five is under the influence of the substance, waves six and seven are recorded after washing out the substance. It was noted that $50 \%$ slowing is reached with both compounds, but in the experiment with the pure substance this value is reached at wave 4 for two retinas and after that 3 retinas were unresponsive to the stimulation, which shows the maximum effect on the excitability of the tissue. The normalization of the spread velocity values permits a better comparison between the outcomes of the two experiments and clearly shows that the model distinguishes between the complex and pure substance. 
(A)
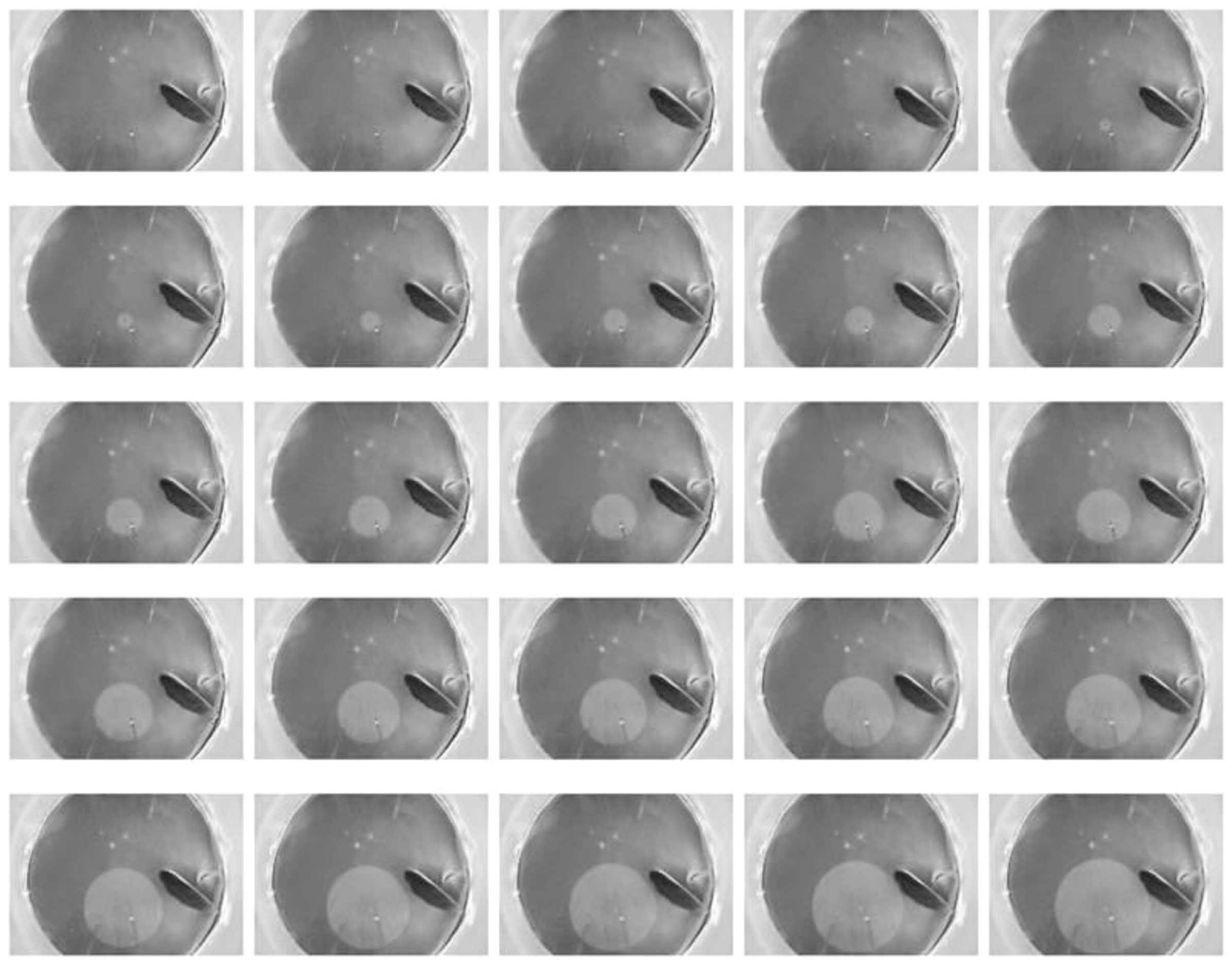

(B)

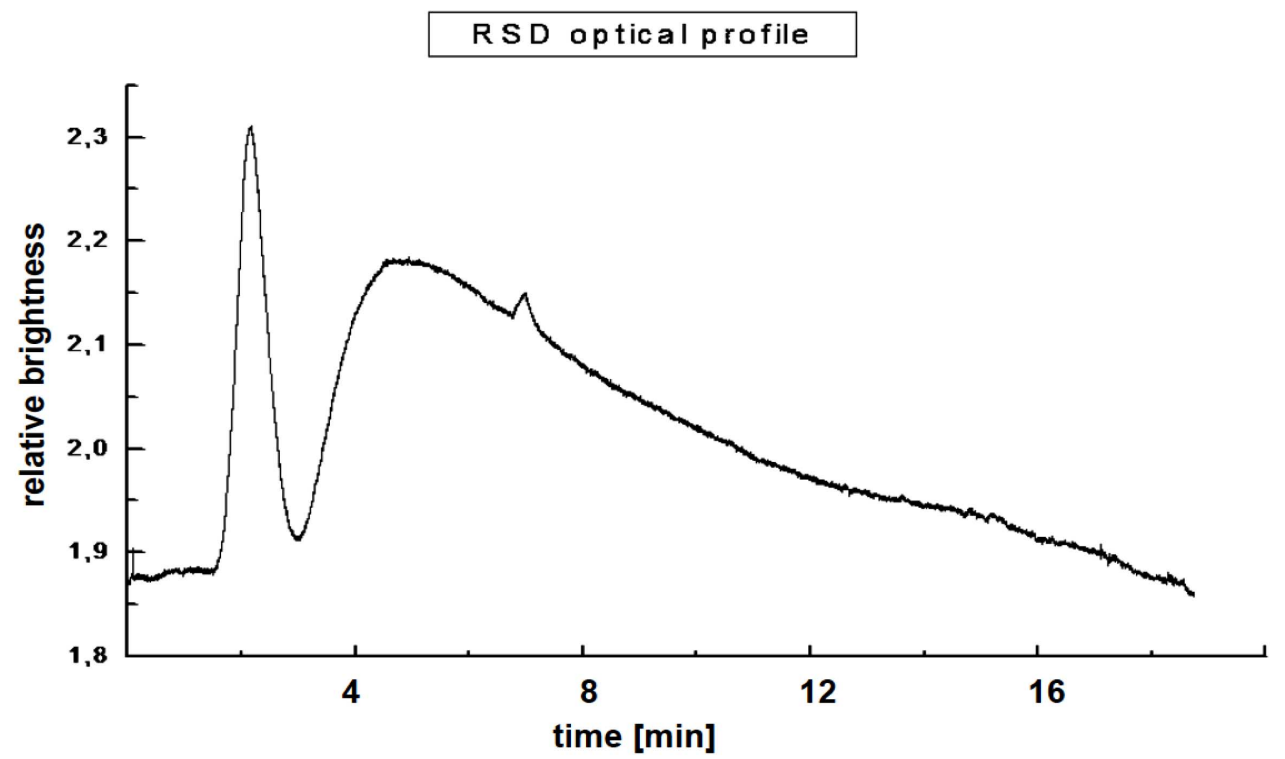

Fig. (1). (A) Sequence of frames showing a mechanical stimulation followed by the development of a RSD wave. From the end of the stimulus to the last frame the time is one minute of wave propagation. The dark structure that is seen inside the eye is the pecten, covered by a dark epithelium and rich in blood vessels. The length of the pecten is $6 \mathrm{~mm}$. The RSD wave evolves with a growing circular pattern of excited tissue. The light scattered by the excited tissue creates the pattern. (B) In part B we show the complete IOS of a retinal wave measured at $29^{\circ} \mathrm{C}$. The advancing first peak of the profile is what is seen in part A of the Fig.. The complete profile duration is 20 minutes. After its completion, tissue refractoriness is over. The profile was measured with a photomultiplier that received the scattered light by the retina from a lens focused at the centre of the eyecup, and that sampled a circular area of tissue of $0.5 \mathrm{~mm}$ in diameter. The photomultiplier voltage output is stored in a computer via an A/D converter with a sampling rate of $5 \mathrm{~Hz}$. The photomultiplier output is expressed in arbitrary units. 


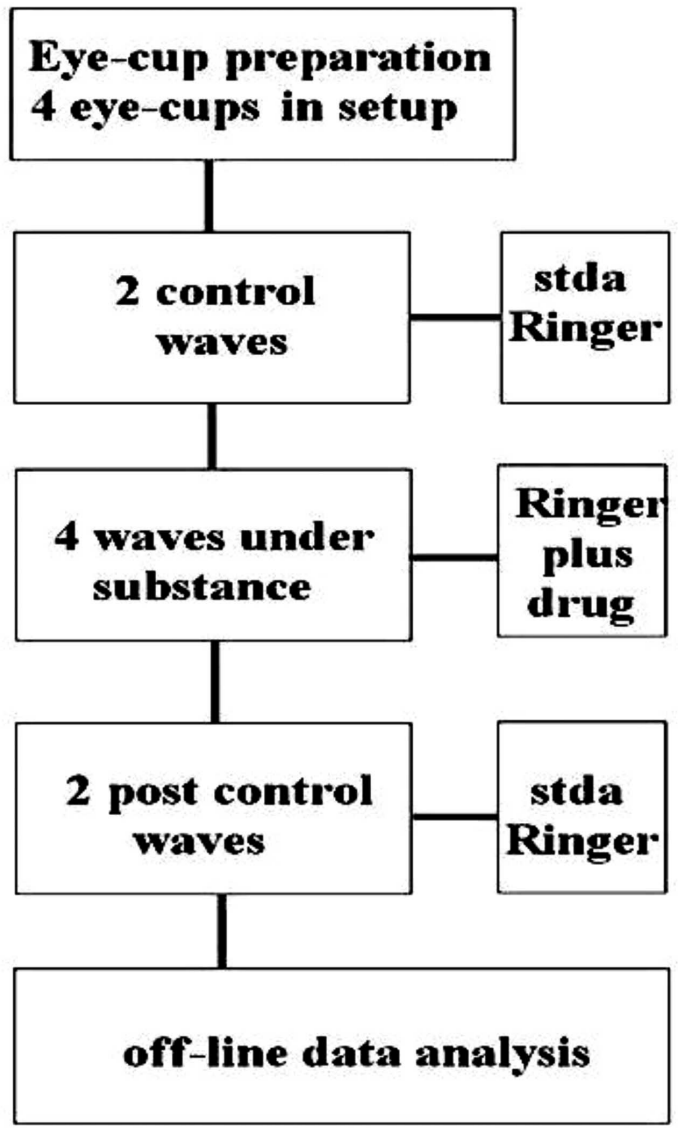

Fig. (2). The flow diagram summarizes the protocol used in the experiments. Isolated waves are elicited mechanically by a light touch in the retina. The spread is observed for 1.5 to $2 \mathrm{~mm}$ and the time for the wave propagation is written down together with the temperature of the Ringer in the dish. Four retinas are stimulated in sequence in each cycle.

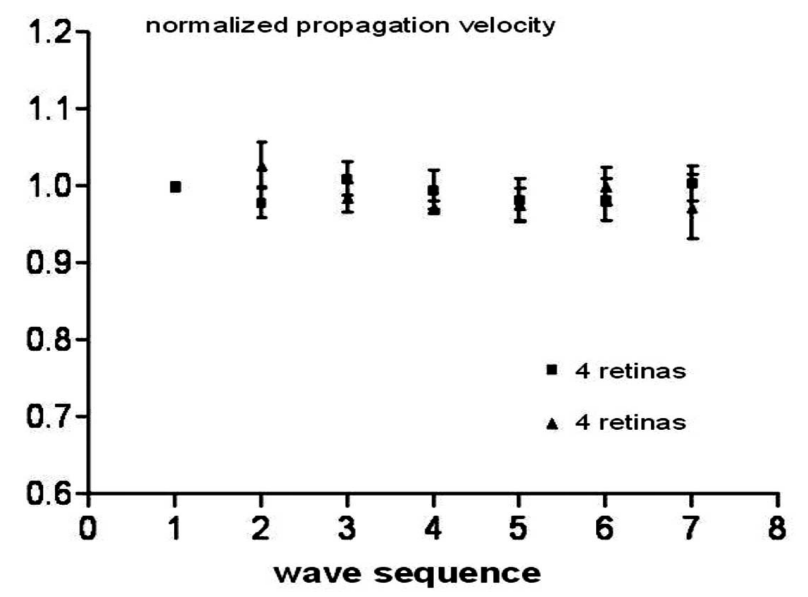

Fig. (3). The graph in the Fig. shows the evolution of the spread velocity of sequential RSD waves elicited mechanically every 20 minutes. The results of two independent experiments each with four retinas are shown to be superimposed in the graph. The normalized mean of the spread velocity and its standard deviations are shown for both experiments. Note the stability of the system response in the standard conditions of the experiments.

The reduction in spread velocity also shows that the modulatory effect of this drug is at the maximum possible, or close to a qualitative change in the system dynamics from excitable state to absolute refractoriness. In Tables $\mathbf{1}$ and $\mathbf{2}$ we show the retinas that became completely refractory, i.e. no RSD wave could be elicited in response to three consecutive mechanical stimuli. For the complex Amlodipine besilate, this maximum effect appears at $70 \mu \mathrm{M}$ concentration, whereas for the Amlodipine standard absolute refractoriness is present at $50 \mu \mathrm{M}$ concentration.
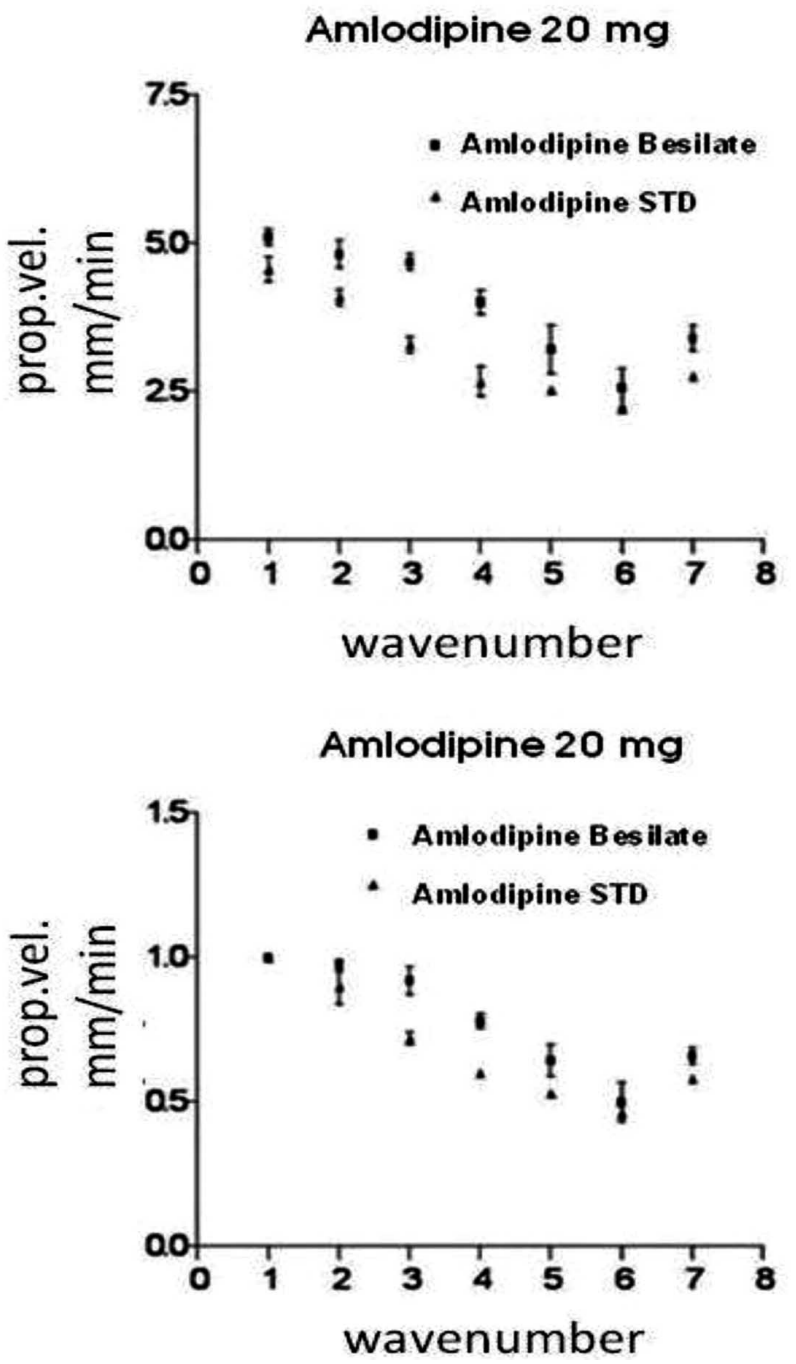

Fig. (4). In the upper part the temporal evolution of the spread velocity of RSD waves is shown for two experiments using the same concentration of Amlodipine besilate and Amlodipine pure substance. At wave number 1 the mean of the measurements for two control waves is shown, waves 2 to 5 were measured in the presence of Amlodipine and waves 6 and 7 during washing out of the substance with standard Ringer in the perfusion system. Note that the two groups of retinas have different spread velocities. In the lower part the same experiments results are shown with normalization of the spread velocities: the control velocity is set to 1 and the other values expressed as percentage of this value. The system response is faster to the pure Amlodipine and thus the system temporal evolution of spread velocity can discriminate between avaiability of the two substances. Four retinas were recorded in parallel in each experiment. The data points show the mean and SD values of the spread velocity for the four retinas. After wave 4, only one retina was responsive to the stimulation in the experiment with pure Amlodipine. 


\section{AMLODIPINE BESILATE $(\mu M)$}

Table 1. The Table Shows the Number of Retinas Responding to Mechanical Stimuli at Each Stimulation Circle.

\begin{tabular}{|l|c|c|c|}
\hline & $\mathbf{5 0}$ & $\mathbf{7 0}$ & $\mathbf{1 0 0}$ \\
\hline \hline 1-Control 1 & $\mathbf{4 / 4}$ & $\mathbf{4 / 4}$ & $\mathbf{5 / 5}$ \\
\hline 2-Control 2 & $\mathbf{4} / \mathbf{4}$ & $\mathbf{4} / \mathbf{4}$ & $\mathbf{5 / 5}$ \\
\hline 3-Wave 2 & $4 / 4$ & $4 / 4$ & $5 / 5$ \\
\hline 4-Wave 3 & $4 / 4$ & $4 / 4$ & $5 / 5$ \\
\hline 5-Wave 4 & $4 / 4$ & $4 / 4$ & $\mathbf{2 / 5}$ \\
\hline 6-Wave 5 & $4 / 4$ & $\mathbf{2} / \mathbf{4}$ & $\mathbf{5 / 5}$ \\
\hline 7-Wave 6 & $4 / 4$ & $4 / 4$ & $\mathbf{5 / 5}$ \\
\hline 8-Wave 7 & $4 / 4$ & $4 / 4$ & $\mathbf{5 / 5}$ \\
\hline
\end{tabular}

Three experiments with 50,70 and $100 \mu \mathrm{M}$ concentration are shown. The cells typed in bold show the number of non-responsive retinas. At $100 \mu \mathrm{M}$, all five tested retinas became non-responsive.

The same protocol can be used to test the presence of the substance in a batch of pills and assess if the response agrees with the stated concentration. Also, the stability of batches over longer time of storage can be tested using the given protocol.

The beta blocker Atenolol HPLC results- We compared the HPLC data of the pure substance Atenolol furnished by industries in Brazil to the Brazilian government standard (Brazil std) and to the secondary European standard (Eu std). Fig. (5) shows a run for the substance supplied by

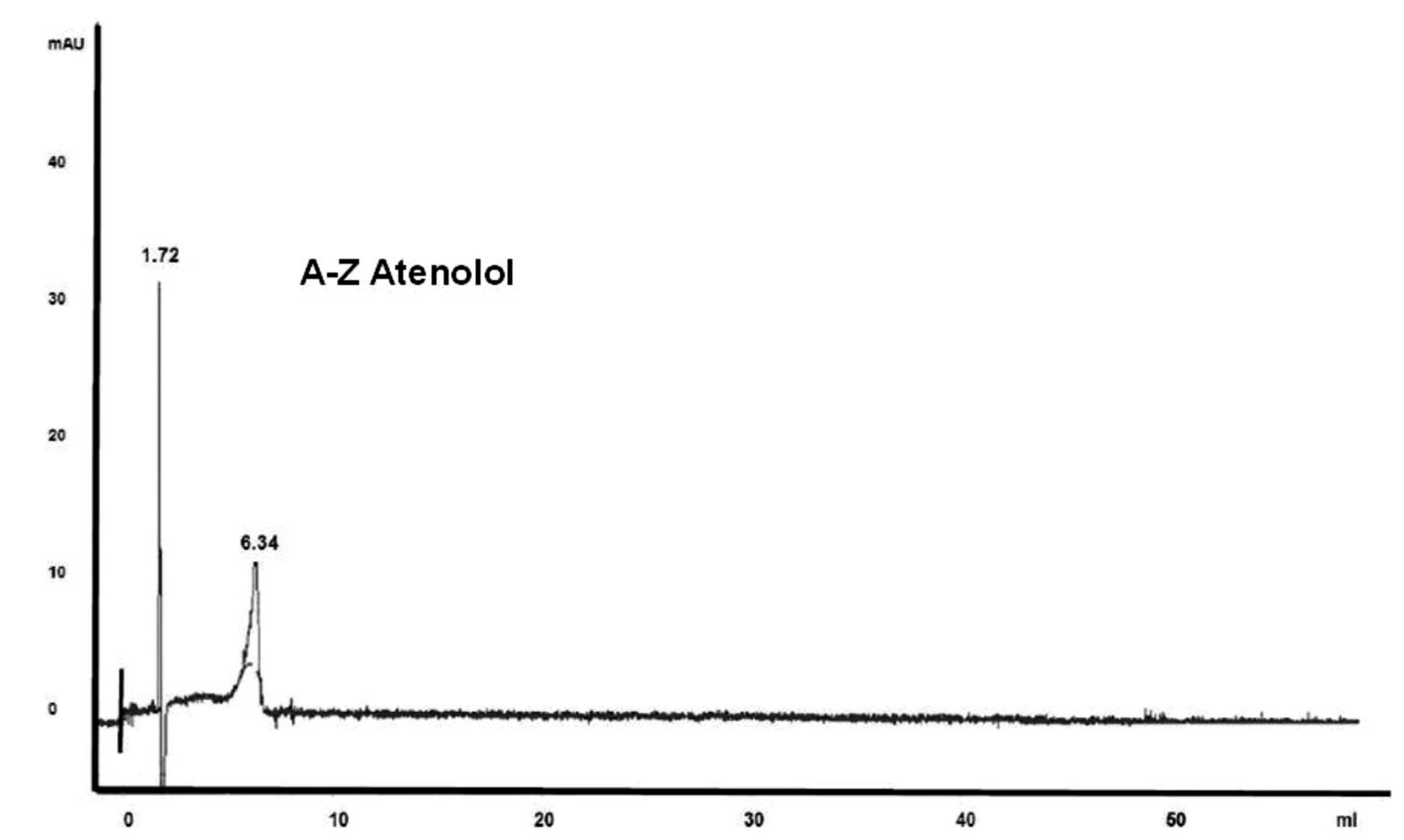

AstraZeneca that sells the reference oral Atenolol preparation in Brazil.

In Table 3 we show the retention time of the main peak and the area under the peak for the European standard, Brazil standard, and EMS that sells a generic Atenolol preparation, Biolabfarma that sells a similar oral preparation, and AstraZeneca. All HPLC curves were similar but they were not identical.

\section{AMLODIPINE EU STANDARD $(\mu \mathrm{M})$}

Table 2. The Table Shows the Number of Retinas Responding to Mechanical Stimulation at Each Stimulation Circle. Two Experiments with 50 and 70 $\mu M$ Concentrations are Shown

\begin{tabular}{|l|c|c|}
\hline & $\mathbf{5 0}$ & $\mathbf{7 0}$ \\
\hline \hline 1-Control 1 & $\mathbf{4} / \mathbf{4}$ & $\mathbf{4 / 4}$ \\
\hline 2-Control 2 & $\mathbf{4} / \mathbf{4}$ & $\mathbf{4} / \mathbf{4}$ \\
\hline 3-Wave 2 & $4 / 4$ & $4 / 4$ \\
\hline 4-Wave 3 & $4 / 4$ & $4 / 4$ \\
\hline 5-Wave 4 & $4 / 4$ & $\mathbf{2} / \mathbf{4}$ \\
\hline 6-Wave 5 & $4 / 4$ & $\mathbf{3 / 4}$ \\
\hline 7-Wave 6 & $\mathbf{2 / 4}$ & $\mathbf{3 / 4}$ \\
\hline 8-Wave 7 & $4 / 4$ & $\mathbf{3 / 4}$ \\
\hline
\end{tabular}

In Fig. (6) we show an HPLC run for the Atenolol extracted from pills of EMS. As expected, the area (related to purity) is smaller and so is the retention time for the main

Fig. (5). The graph shows an HPLC run made with pure Atenolol provided by AstraZeneca. The ordinate shows the relative UV absorption at $225 \mathrm{~nm}$. The numbers at the top of the peaks show the retention times in minutes. The abscissa shows the amount of buffer in ml. The first peak at 1.72 is the solvent (acetonitril/water). 


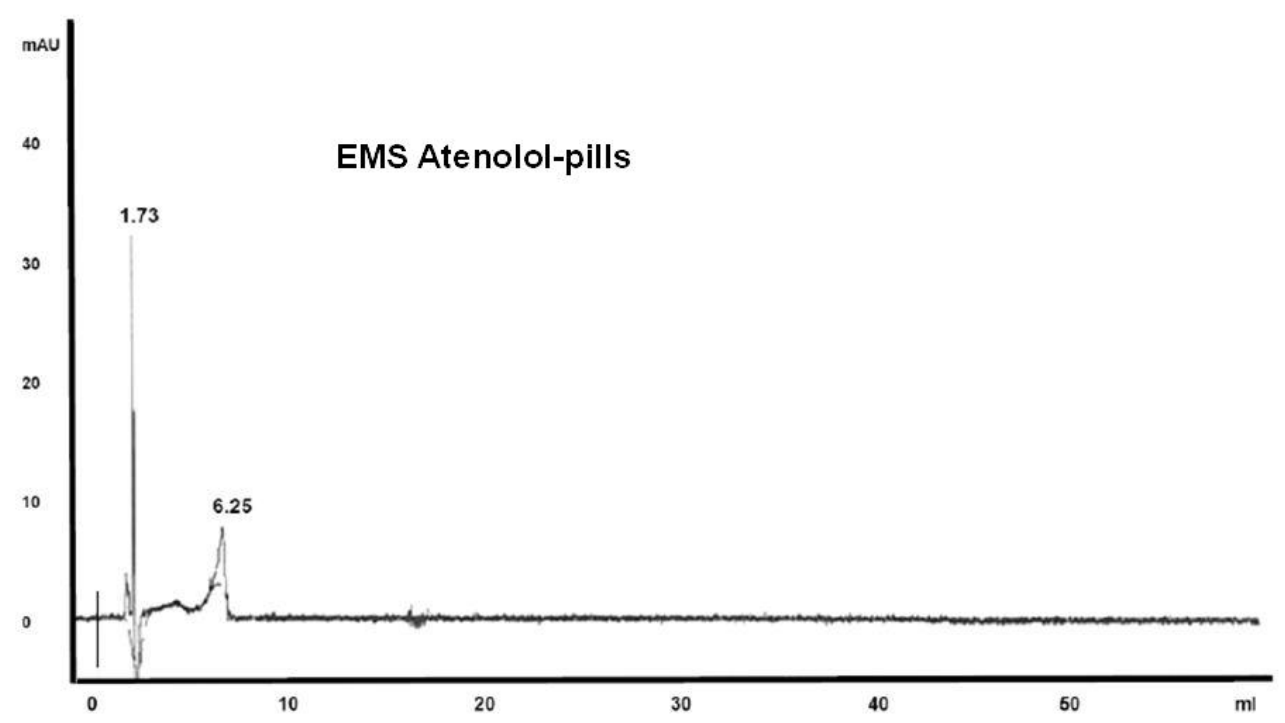

Fig. (6). The graph shows an HPLC run made with Atenolol extracted from pills of the generic oral preparation from EMS. Otherwise the Fig. is identical to the previous Fig.. Note the similarity of the graphs.

peak. The amount of active principle in the pills was measured for the reference preparation and for two generic preparations. AstraZeneca had $25.0 \mathrm{mg}$ per pill (100\% nominal value); EMS $24.4 \mathrm{mg}$ (97.6 \% nominal value) and Biosintética 24.2 (96.8\% nominal value).

Table 3. The Table Shows the Retention Times and are Under the Main Peak of the HPLC Runs for the Different Sources of Atenolol Pure Substance and the Runs Made with the Atenolol Extracted from the Oral Preparations of EMS

\begin{tabular}{|c|c|c|c|}
\hline Substance & $\begin{array}{c}\text { Mean Retention } \\
\text { Time }\end{array}$ & $\begin{array}{c}\text { Mean Peak } \\
\text { Area }\end{array}$ & $\mathbf{N}$ \\
\hline \hline Europ. STD & 6.88 & 5.30 & 3 \\
\hline Brazil STD & 6.76 & 5.31 & 3 \\
\hline EMS & 6.74 & 6.83 & 5 \\
\hline Biolab & 6.57 & 4.33 & 5 \\
\hline AstraZeneca & 6.36 & 3.49 & 3 \\
\hline EMS pill Lot: 038177 V1005 & 6.26 & 2.12 & 2 \\
\hline
\end{tabular}

The retina system response to Atenolol- The Atenolol molecule has a chiral center with stereoisomers. The enantiomer effect was measured comparing the racemic mixture present in the oral presentations with the pure left and right isomers. In Fig. (7), the results of the perfusion with Atenolol, $100 \mu \mathrm{M}$, on the spread velocity are shown for waves obtained after 90 minutes of perfusion with the drug dissolved in Ringer solution. The bars show the mean and $\mathrm{SD}$ of the distribution of values for 4 retinas. The difference in the means of Atenolol+ and Atenolol- compared to the control and Atnolol were statistically significant at $\mathrm{p}<0.02$ level (unpaired t-test). Thus, a difference in chirality can influence the retinal system response to a drug. In a world where there are literally thousands of companies are competing to sell active principles, this result can be relevant.

\section{action of $100 \mu \mathrm{M}$ atenolol after $90 \mathrm{~min}$}

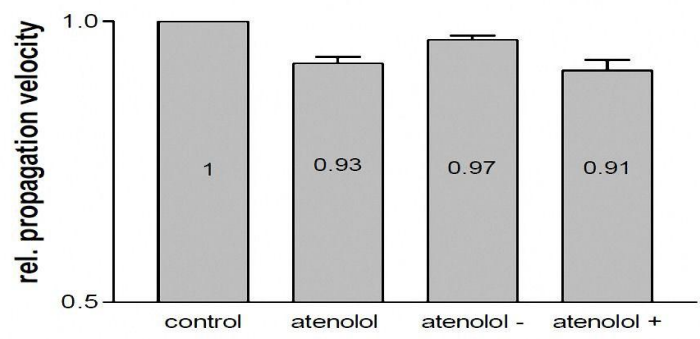

Fig. (7). The bars in the graph show the mean and SD of normalized spread velocities of RSD waves measured after 90 minutes of perfusion with Ringer in which Atenolol dissolved in DMSO was added. Four retinas were recorded in parallel in each experiment. The difference in the means is statistically significant at $\mathrm{p}<0.02$ level (unpaired t-test)

In Fig. (8) the retinal response to Atenolol is shown for three different samples of pure substance from Astrazeneca and EMS. The superimposed curves are very close and thus statistically indistinguishable. The combined outcome of the three experiments in each case is shown to be superimposed on the experiment made with the Atenolol Brazilian standard. The retina results and the HPLC results are then comparable in sensitivity.

The retina system response to oral preparations of Atenolol- The bioavailability of the hydrophobic Atenolol was increased by applying DMSO before dissolution in the retina Ringer. At the concentrations used, the dispersant does not affect the system response. The formulations of all oral preparations sold in Brazil use sodium lauryl sulfate (SDS) as dispersant. The other inert ingredients of the pills: 


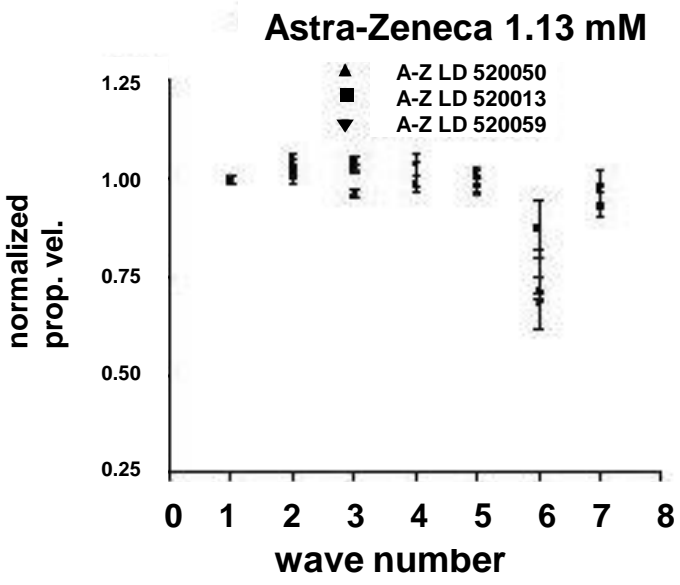

EMS Comp. $150 \mathrm{mg}$

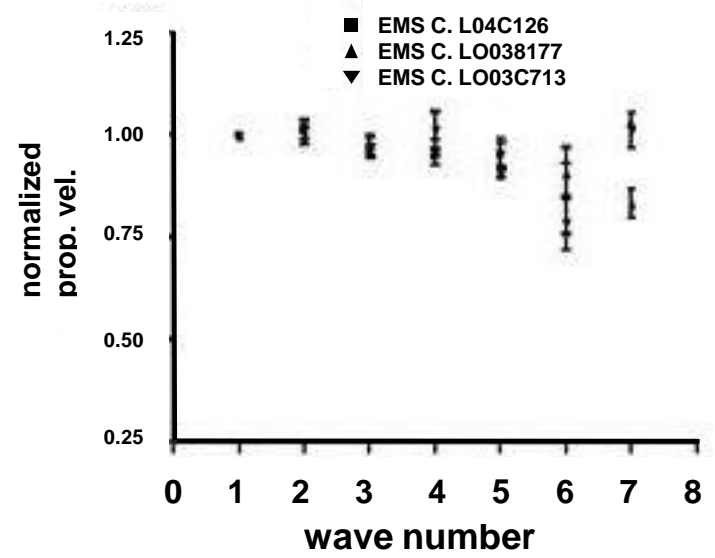

Brazil STD vs. A-Z $1.13 \mathrm{mM}$

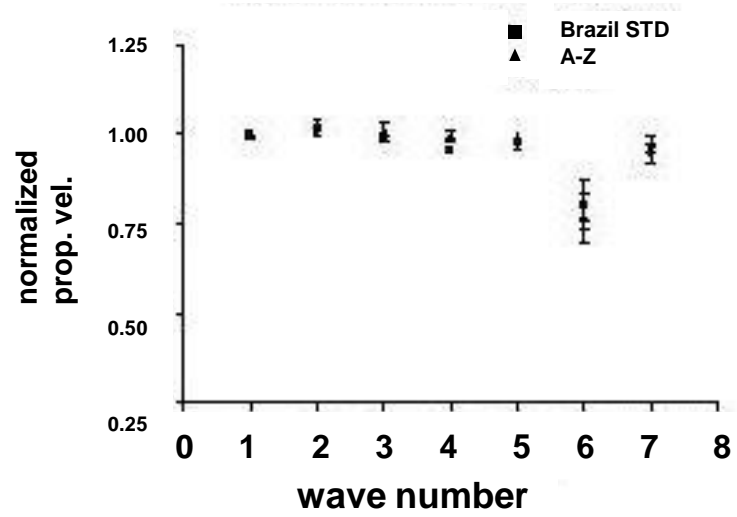

Atenolol $1.13 \mathrm{mM}$

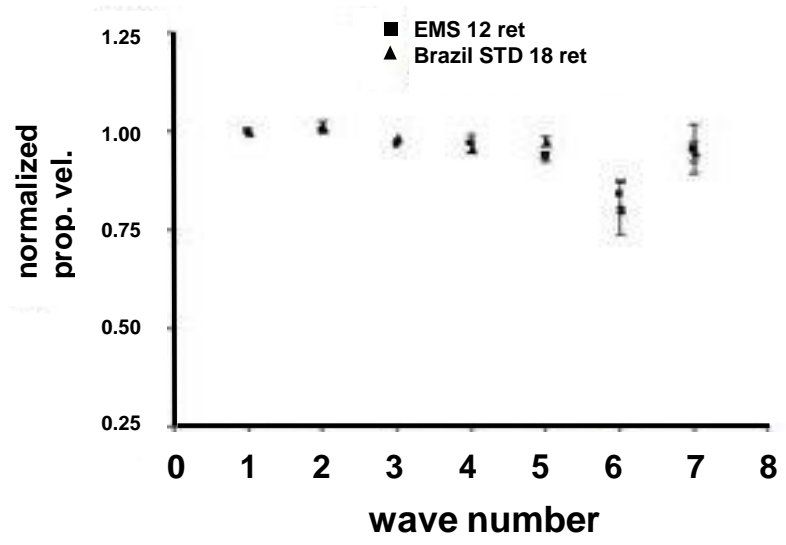

Sigma vs. Brazil std.

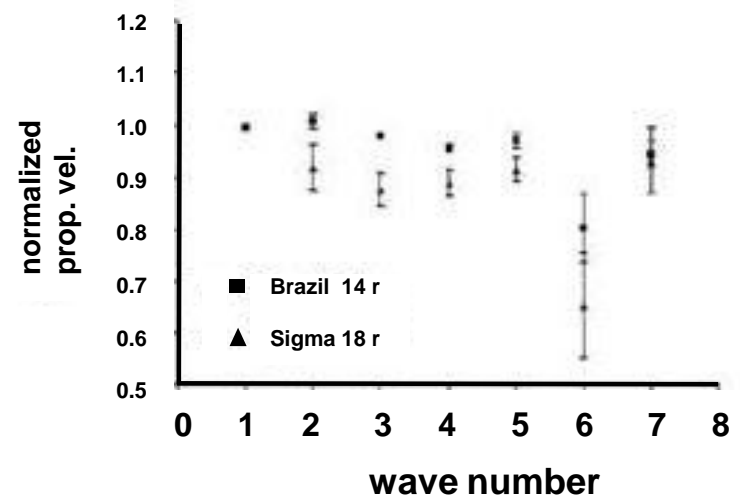

Fig. (8). First row: the left graph shows the system response to three batches of pure Atenolol from AstraZeneca. The data from the three experiments are superimposed. In each experiment four retinas were recorded in parallel. On the right we compared the outcome of the 12 retinas with the system response to Atenolol Brazil standard. Second row: the left graph shows the system response to three batches of Atenolol from EMS. On the right the combined outcome of the 12 retinas is compared to the system response to Atenolol Brazil standard. Third row: the graph shows the system response to Atenolol Sigma laboratory grade and to the Atenolol Brazil standard. All experiments used the same concentration of Atenolol.

magnesium stearate, magnesium carbonate (heavy), starch and gelatin would either be separated by the centrifuge or they are not interfered with the measurements. In Fig. (9) we show the retina system response to Atenolol extracted from oral preparations for the reference compound (AstraZeneca) and the generics from EMS and Biosintética. 

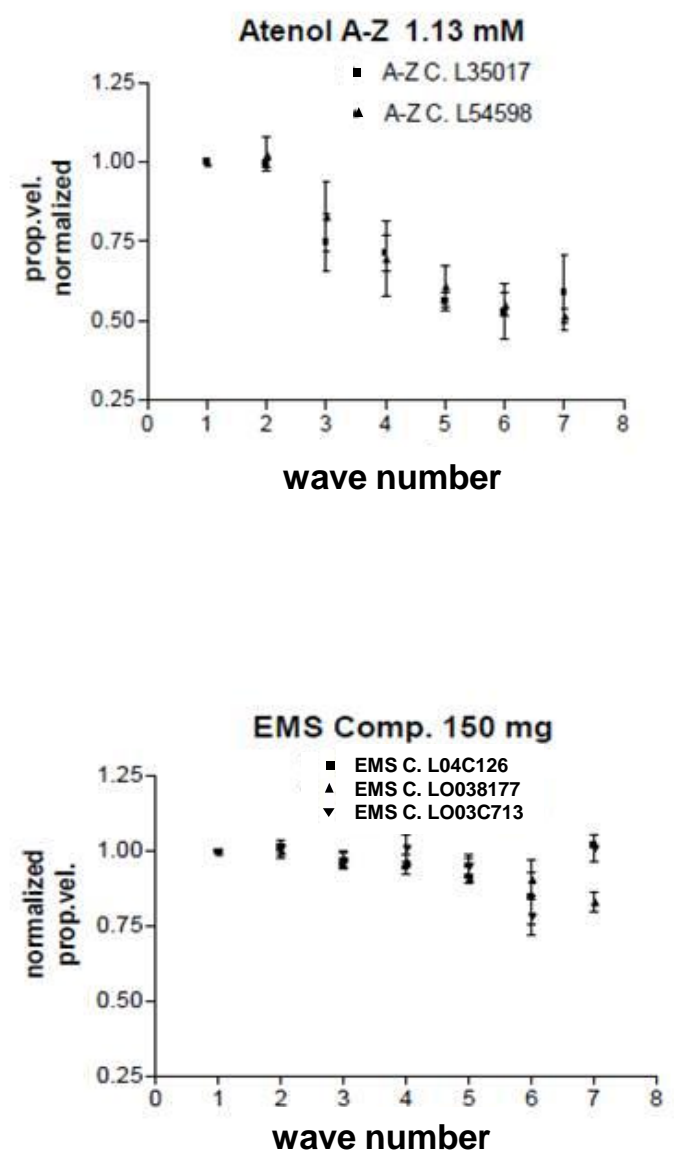
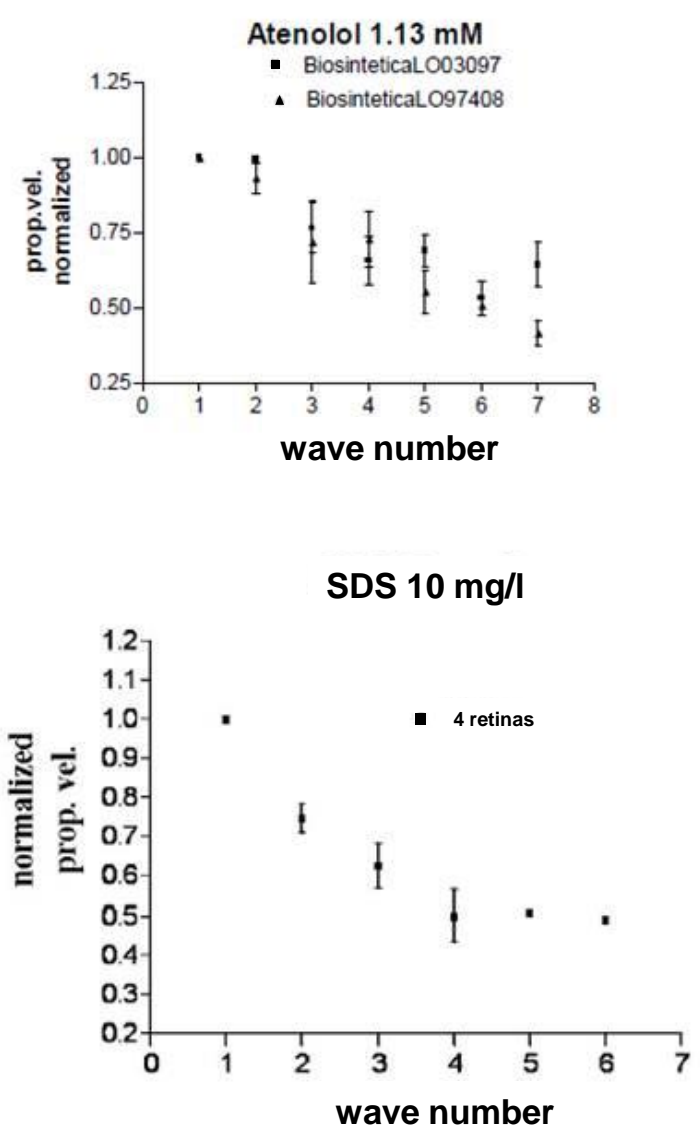

Fig. (9). The graphs show the system response to Atenolol extracted from oral preparations. The concentration was the same in the experiments. Note that for AstraZeneca and Biosintética the response are similar and differ from the experiments with pure substance Atenolol. For the Atenolol extracted from EMS pills, the system response is very similar to the pure substance response. The response of two or three batches of pills are superimposed. On the graph labelled SDS, we show the system response to sodium lauryl sulphate alone added to the Ringer.

The behavior of the EMS extract is qualitatively different from the reference and it is very similar to the application of the pure active principle as shown in the previous figure. We decided to test the effects of SDS alone in retinas and its interaction with the active principle Atenolol in pure form (using the Brazilian standard). The results are shown in Fig. (10). It should be noted that there is no effect a $2 \mathrm{mg} / \mathrm{l}$ and a very strong excitability modulation at $10 \mathrm{mg} / 1$. The addition of SDS at $2 \mathrm{mg} / 1$ to Atenolol increased the Atenolol effect (from 5 to $9 \%$ reduction) on the spread velocity of RSDs.

Next, we proceeded in measuring the amount of SDS per pill in several oral preparations sold either as generics or similar oral preparations. The upper and lower parts of Fig. (11) show these results. The one way ANOVA technique applied to the data shown in Fig. (11, lower part) gave an F value of $69.55 p<0.01$. The variability in batch by batch in the different oral preparations was also noted. The reference pills from AstraZeneca and the similar of Biolab-Farma had the smaller variations, whereas Biosintética had the biggest followed by EMS. The concentration in the EMS pills was

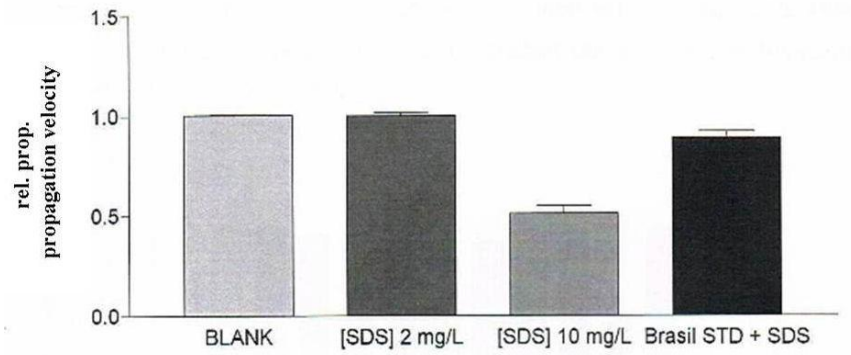

Fig. (10). The bar graphs show the effect of SDS and SDS $2 \mathrm{mg} / \mathrm{l}$ plus Atenolol pure substance 1,13 mM Brazil STD. $\mathrm{N}=4$ retinas for the SDS experiments and 6 retinas for the SDS plus Atenolol. Note the lack of effect at $2 \mathrm{mg} / \mathrm{l}$ and the strong effect at $10 \mathrm{mg} / \mathrm{l}$. The addition of SDS $2 \mathrm{mg}$ to Atenolol had a small effect that was not statisitically significant when compared to results of just pure Atenolol.

so small that it affected the bioavailability of the active principle. The retina system findings agree with the values presented to ANVISA for the pharmacokinetics results of 

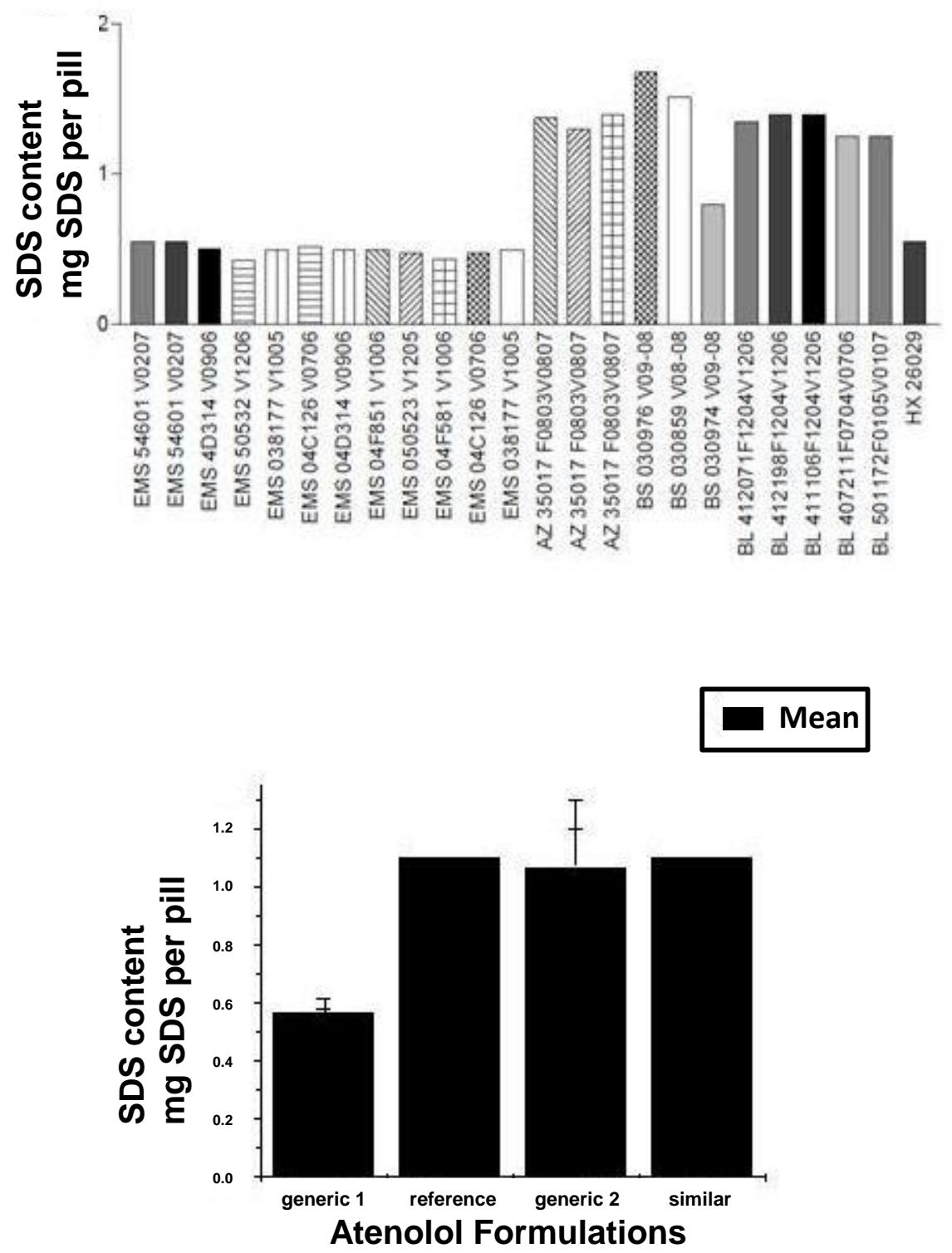

Fig. (11). Upper part: the bars show the amount of SDS found in individual pills from different batches from EMS, AstraZeneca (AZ), Biosintética(BS), Biolab (BL) and Hexal. Note that EMS has the least amount, Biosintética the largest variation and Biolab and AstraZeneca have similar concentration of SDS and have the smallest variation. Lower part: The results of the individual batches are combined for EMS (generic1), AstraZeneca (reference) and Biolab (similar).

AstraZeneca, EMS and Biosintética that were respectively, $1.5,2.5$ and 1.5 hours for the peak time of the plasma concentration in humans. If the criterion of maximum $20 \%$ difference in the peak time is used, then EMS oral preparation differs from the reference. However, one can remember that bioequivalence and therapeutic equivalence are two different concepts in pharmacology. We can conclude that the in vitro retina system displays high sensibility and reliability, and could be the basis of a robust methodology for the creation of pharmacological data banks within intelligent systems with automatization of recording protocols and output.

Finally, it is interesting to compare the modulatory effects on the excitability of gray matter of Atenolol with the beta blocker Propanolol. Propanolol is a very effective modulator of tissue excitability reaching the maximum possible effect (50\% slowdown of spread velocity) at 400 $\mu \mathrm{m}$ concentration in the bath solution [18]. Atenolol development was justified on the grounds of better affinity for the heart receptors. It is reasonable to propose that the therapeutic effects of Propanolol in migraine could be more related to the modulatory effect measured in retina experiments (intact tissue) and that these effects could plausibly be only indirectly related to ligand receptor properties or even be due to other properties of the therapeutic molecule.

\section{CONCLUSION}

The retinal spreading depression combined with modern data-basis technology is a useful tool for quality control and 
validation of the action of new drugs in pharmacological research when used to complement cellular and animal experiment by tissue experiments. It is cost efficient, easy to handle, and delivers relevant results in short time.

\section{ACKNOWLEDGEMENTS}

None declared.

\section{CONFLICT OF INTEREST}

None declared.

\section{ABBREVIATIONS}

$\begin{array}{ll}\text { ANVISA } & =\text { National Agency for Sanitary Vigilance } \\ \text { CNS } & =\text { Central nervous system } \\ \text { IOS } & =\text { Intrinsic optical signal } \\ \text { IT } & =\text { Information technology } \\ \text { RSD } & =\text { Retinal spreading depression } \\ \text { SDS } & =\text { Sodium-dodecyl-sulfate }\end{array}$

\section{REFERENCES}

[1] Wiedemann, M.; Pieffel, A.; and Hanke, W. Effects of different gravity conditions on self-organizing biological systems. Signal Transduction, 2006, 6, 414-421

[2] Wiedemann, M.; Kohn, F.P.M.; Roesner, H.; Hanke, W.R.L. Selforganization and pattern formation in neuronal systems under conditions of variable gravity., Springer, New York, 2010

[3] Gotti, C.; Moretti, M.; Maggi, R.; Longhi, R.; Hanke, W.; Klinke, N.; Clementi, C. Alpha7 and alpha8 nicotinic recreptor subtypes immunopurified from chick retina have different immunological, pharmacological and functional properties. Eur. J. Neurosci., 1997, 9(6), 1201-1211

[4] Vailati, S.; Hanke, W.; Bejan, A.; Barabino, B.; Longhi, R.; Balestra, B.; Moretti, M.; Clementi, F.; Gotti, C. Functional alpha6containing nicotinic receptors are present in chick retina. J. Mol. Pharmacol., 1999, 56(1), 11-19
[5] Marques, J.A.H. Depressão alastrante em retinas de mamiferos. MSC Thesys, Instituto de Biofísica, UFRJ, 1977

[6] Martins-Ferreira, H. Depressão alastrante na retina in vitro. An Acad. bras. Cienc., 1962, 34(4):XLIV-XLV.

[7] Martins-Ferreira H.; de Oliveira Castro, G. Light scattering changes accompanying spreading depression in isolated chicken retina. J. Neurophysiol., 1966, 29, 715-726.

[8] Fernandes de Lima, V.M.; Hanke, H. Excitation waves in Central Grey Matter: the Retinal Spreading Depression, Prog. Retin. Eye Res., 1997. 16(4), 657-690.

[9] Hanke, W.; Fernandes de Lima, V.M. Central nervous tissue: an excitable medium. A study using the retinal spreading depression as a tool. Philos. Transact. A Math. Phy. Eng. Sci., 2008, 366, 359368

[10] Dowling, J. The Retina: an Approachable Part of the Brain, Harvard University Press: Cambridge, USA, 1987

[11] Fernandes de Lima, V.M.; Piqueira, J.R.C.; Hanke, W. The synergetic modulation of the excitability of central gray matter by a neuropeptide: two protocols using excitation waves in chick retina. An. Acad. Bras. Cienc., 2009, 81(1), 1-11.

[12] LAFIS- Industria Farmaceutica. Available at: www.lafis.com.br/ institucional/relatorio-analise-setorial/Industria-Farmaceutica.asp?f $=$ as, 2011

[13] Fardelone, L.; Branchi, B.A. Recent changes in the pharmaceutical market. Rev FAE Curitiba, 2006, 9(1), 139-152. Available from: http://www.fae.edu/publicacoes/pdf/revista_da_fae/fae_v9_n1/rev_ fae_v9_n1_10_lucidio.pdf

[14] Andrade de Oliveria, E.; Labra, M.E.; Bermudez, J. Public production of medicines in Brazil: an overview Cad. de Saúde Pública Rio de Janeiro, 2006, 22(11), 2379-2389

[15] Weimer, M.; Hanke, W. Correlation between the durations of refractory period and intrinsic optical signal of retinal spreading depression during temperature variations. Exp. Brain Res., 2005, 161, 201-208.

[16] Brand, S.; Dahlem, M.; Fernandes de Lima, V.M.; Muller, S.C.; Hanke, W. Dispersion relation of spreading depression waves in chicken retina. Int. J. Bifurcat. Chaos, 1997, 7(6), 1359-1365

[17] Brand, S.; Fernandes de Lima, V.M.; Hanke, W. Pharmacological modulation of the refractory period of retinal spreading depression. Naunyn Schiemedebergs Arch. Pharmacol., 1998, 357, 419-425

[18] Wiedemann,.M.; Fernandes de Lima, V.M.; Hanke, W. Effects of anti-migraine drugs on the retinal spreading depression. Naunyn Schiemedebergs Arch. Pharmacol., 1996, 353, 1-5. 